\title{
Basic Tips for Communicating with A New Dental Patient
}

\author{
Antoniadou Maria ${ }^{1}$, Kitopoulou Anna ${ }^{2}$, Kapsalas Andreas ${ }^{2}$, Tzoutzas Ioannis ${ }^{3}$ \\ ${ }^{1:}$ Assistant Professor, ${ }^{2:}$ Dentist, ${ }^{3:}$ Professor \\ Department of Operative Dentistry, Dental School of Athens, \\ National and Kapodistrian University of Athens, Greece.
}

\begin{abstract}
The process of treating a dental patient requires the dentist to possess several skills along with high technical expertise. Good communication skills guaranties trust and long lasting cooperation while at the same time improve patient's compliance and overall satisfaction. There are certain basic principles of practicing good communication and proceeding in an effective interviewing with a new dental patient. Usually two appointments are required for this purpose. Effective communication in such a case includes nonverbal ways, engaged listening, managing stress in the moment, the ability to communicate assertively and the capacity to recognize and understand one's own emotions. Overall, the initial dentist-patient communication should be reinforced by empathic behavior from the part of the dentist. Effective communication tips can be learned and practice despite patient's cultural background. In this article an analysis of the basic communication knowledge, is made knowledge that is important for the two first appointments with a new dental patient.
\end{abstract}

Keywords: Dentist-patient communication, body language, interviewing a patient

\section{INTRODUCTION}

Knowledge and technical skills are not the only prerequisites for a successful dental practice. The ability to communicate effectively with patients was always of outmost importance and need for such a success [1,2]. Nowadays, with the global economic crisis within multi-ethnic societies, is even more necessary for the dentist to be able to communicate patient's needs while respecting the economical survival of his practice.

Communication is in generally more than just exchanging information. It's about understanding the emotion and intentions behind the information given in any circumstance. It has two sides at the level of the dentist-patient communication: the task-focused, cure-oriented one that deals with the practical things of the treatment and the affective, socio-emotional, care-oriented part that targets at the overall care [3]. Of course, the effective communication process is always a two-way street. It's not only how a message is received and understood by someone in exactly the way it was intended to, but it is also how someone listen to another person, in order to gain the full meaning of what is being said and simultaneously to make the other person feel heard and understood $[4,5,6]$. So the effective communication is not consisted only of verbal expressions but combines a set of skills including nonverbal communication, engaged listening, managing stress in the moment, the ability to communicate assertively and the capacity to recognize and understand one's own emotions as well as those of the person with whom the communication takes place [7].

Communication skills in the dental practice influence patient's decision to select therapeutic plans and even remain in the practice by deepening interpersonal connections, improve teamwork, decision making and problem solving [8]. Additionally, they increase patient's satisfaction since this satisfaction depends not only on the clinical skills of the clinician, but also on his/her non-verbal behaviors, such as body expressions [6,8,9], one's own personality and it even enables dentist to communicate negative or difficult messages without creating conflict or destroying trust [10]. Moreover, effective communication can result in building a trusting patient-dentist relationship and has therapeutic effect on patients, who are more likely to decide to follow the treatment plan suggested by the dentist [2]. It has been proven that patients have more confidence in dentists who have the ability to communicate care and compassion above all other positive characteristics [11]. Although a lot of data exist on the verbal and non-verbal communication skills needed for an effective 
business transaction/relationship [12,13], little have been discussed on the way the dentist as a professional should react, speak or react with his body language in the first appointment with a new dental patient $[14,15,16]$. The dental patient is usually overflowing with fear, pain, agony, insecurity and anxiety [17]. These emotions contribute to a less than ideal ground for an effective communication. Thus in this paper an attempt to suggest evidence based attitudes during the first two introductory appointments with a new dental patient, is made.

\section{Challenges of the dental communication field}

Achieving effective communication in the dental practice includes many challenges due to the sentimental situations involved while sitting in the dentist's waiting room. Furthermore, it is now well established that patients' recall of a medical consultation is somehow limited anyhow, with patients forgetting 40-80\% of the information given [18]. It should be then noted that an overestimation of an effective result from an interview with a new patient, is not whatsoever wise, since another relevant study has shown that although $75 \%$ of a team of orthopedic surgeons believed that they communicated in a satisfactory way with their patients, only $21 \%$ of their patients reported relevant satisfactory communication with the doctors [19]. Usually, dentists' recall of a discussion is greater than that of patients. Dentists overall recall more activities taking place in the consultation phase, like more oral health advice that are being offered and more discussion of follow-up actions. On the other hand, patients are particularly poor at recalling dental health advice and future actions relating to consultation [18].

It is supported that patients seem to appreciate dentist's empathy. Empathy is the capacity to understand or feel what another person is experiencing from within the other being's frame of reference, i.e., the capacity to place oneself in another's position [20]. So patients report as positive the dentist's care and concern for their oral health, when these are provided with clear instructions and explanations and when support staff members are friendly and helpful. It seems that the most important parts of dentist-patient communication are: a) treating patients as individuals with unique needs and concerns (not a "mouth"), b) helping to teach them how to take care of their mouth without lecturing, c) not continuing with the procedure if patients are in pain and d) making sure that they are aware of treatment costs prior to beginning of the therapy [11,21].

Furthermore, patients have some common expectations from their physicians, which are divided into primary expectations, such as clinical competence and secondary expectations, such as being professional, respectful, polite, sincere, interested and effective in communication [4,5]. In other words, increased patient satisfaction is influenced not only by the physician's clinical skills but also by his non-verbal behavior, active listening, the ability to decode body movements and the ability to adapt to patients' expectations and specific emotional status which is referred to fear and pain while sitting at the dental office [6,22]. All relevant data though suggest that doctor's and patient's interpersonal communication is generally, one of the most complex forms of communication, for the following reasons: 1) it is a communication established by rules and conventions of a particular type of activity, 2) it is a communication between non-equal partners, 3) it is not spontaneous, 4) its key aspects are significant and therefore can be emotional, 4) it requires close cooperation $[3,5,6]$. For all these reasons, learning of certain communication keys seems absolutely necessary for the dental professional.

\section{Verbal and non verbal communication}

Communication can be both verbal and nonverbal [23,24]. It is said that non-verbal communication developed before verbal communication. For this reason people have learned first to react to non verbal messages [3]. Verbal communication involves exchanging messages using words or language. Along with words and language, verbal communication includes tone and volume of the voice [25]. Verbal communication includes speaking and listening skills. Speaking is part of the everyday communication, thus the choice of words can make a big difference in whether a message is understood and how it is received. Listening is an important part of the communication process [26]. Active listening is a form of listening that involves giving full attention to the sender and to the message that he communicates $[25,27]$.

Non verbal communication includes [3]: 1) Kinesics or body language, that refers to body movements, especially for face and hands. Also refers to the body attitude (standing or sitting), 2) Proxemics, that refers to the usage of space, how far the communicating people are standing, 3) 
Haptics, which refers to the touch as a communicating way, especially type, frequency and intensity and 4) Chronemics, which refers to the usage of time and includes accuracy in hour turnout in professional or social occasions.

Non verbal communication occurs without words and can show thoughts and inner feelings. It accounts for over $95 \%$ of the first impression we have on others [5,28]. In the first seconds of a new accountancy, amygdale, an almond-shaped section of nervous tissue located in the temporal (side) lobe of the brain, which is responsible for the perception of emotions such as anger, fear, and sadness, as well as the controlling of aggression [29], is addressing two main questions: a) "What are this person's intentions towards me?" and b) "How strong and competent is this person?". These two questions establish the first base of human communication. Then, non-verbal communication with eyes, hands, feet and overall position of the body, incorporates more information on the character of the doctor as well as that of the patient. For example, making eye contact is one of the most basic forms of non verbal communication. Looking someone in the eyes can communicate feelings of interest, attention, respect or negative ones like hate. On the other hand, avoiding eye contact can communicate shyness, lack of attention, boredom or disrespect. Furthermore, many body signals represent different meanings depending on the person and the situation $[12,28]$. For example, if a patient clears its throat during a conversation it could be interpreted as anxiety, but it can also mean that she/he simply has a sore throat or it is just a habit [9]. For that reason it is important to understand and know how to interpret patient's body language.

One main aspect that controls body language for both dentist and the patient is the stress $[23,30]$. Learning to manage stress in the heat of the moment is one of the most important things a dentist can do in order to improve his non verbal communication. Stress compromises the ability to communicate and leads to misreading other people, send confusing or off-putting non verbal signals, and lapse into unhealthy knee-jerk patterns of behavior $[12,16]$. Furthermore, stress, like other emotions, is contagious. If the dentist is upset it is very likely to trigger others to be upset too, making a bad situation even worse.

Naturally, the process of understanding body language needs preparation and practice [13]. In the first meeting there is a need to evaluate external appearance, body language and emotions of the patient. Elements of the external appearance, such as dressing, hair color, jewelry and tattoos are also part of non verbal communication [3]. For example does he/she wear clothes suitable for a doctor's appointment? The dentist can start to illustrate a general picture for the person standing across from us from external characteristics, because they say a lot about how a person wants to be seen.

Also people from different countries and cultures tend to use different non verbal communication gestures, so it's important to take age, culture, religion, gender, and emotional state into account when reading body language signals. A European teen, a grieving widow and an Asian businessman, for example, are likely to use non verbal signals differently. Finally, the dentist should look at non verbal communication signals as a group. He should not consider a single gesture or non verbal cue, but all of the non verbal signals he receives, from eye contact to tone of voice to body language. Anyone can slip up occasionally and let eye contact slip, for example, or briefly cross their arms without meaning to. The dental professional should consider the signals as a whole to get a better "read" on a person. The non verbal communication should reinforce what is being said, not contradict it. If someone says one thing, but the body language says something else, the listener will likely feel that the speaker is dishonest. For example, no one should say "yes" while shaking the head "no". Furthermore, in order to analyze and understand a patient's body language the dentist should focus on some standard behavior and evaluate any alternation from that, during the appointment $[2,31]$. In the end, the dentist should always analyze the characteristics he sees as a whole, like putting together the pieces of a puzzle $[3,13]$.

At the same time, during the conversation with a patient, the dentist should be aware of his own body posture, and what signals he personally may give [31]. This first impression is crucial and important to create a friendly and approachable first image. For example, a clean, elegant, comfortable and contemporary place, where everything is tidy, and there is also some relaxing music that are combined with a warm and sincere smile and a warm handshake create a good first impression and help significantly in the subsequent communication $[3,32]$.

Body language displays sincerity. The goal is to stimulate conversation and encourage follow-up 
questions [2,11]. This goal can be achieved by: 1 ) looking up frequently and making eye contact with the patient, nodding and engaging them 2) having a face that is emotionally present and filled with interest 3) speaking with a smooth voice that projects warmth, confidence and interest 4) presenting a body that is open to communication and relaxed, 5) respecting one's own personal space by staying one arm's length away from the patient. In general, non verbal communication requires background skills such as audience awareness, personal presentation and body language management.

\section{DENTAL INTERVIEW}

The initial interview of a new dental patient should be divided in two appointments as follows:

\section{$1^{\text {st }}$ appointment: interviewing the patient}

\section{First part: Meeting the patient}

When meeting the patient for the first time in the waiting room, a warm welcome should be addressed from the dentist to the patient potentially, escorted with a vertical type typical hand-shake. The professional should then introduce himself to the patient with his official birth name. Of course, it is important to know beforehand the name and some details about the patient before he walks towards the patient. It is suggested to never call a patient, by his/hers first name in the first appointment, except of the pediatric age patient. In any case the dentist should always ask permission for addressing the patient in an informal way [23]. The patient should then be directed into the office, following the dentist who shows the way. Meanwhile, the dentist should always make eye contact with the patient in a professional, polite and personal way [31]. This part of the interview is suggested to last no more than two minutes. It will be helpful if communication starts at this point with a neutral theme of discussion like the weather (for example: It's been lovely today.. Or it is raining today..are you wet? etc.). Of course, it is widely suggested to never discuss on politics, religion and/or sex $[12,15,25]$.

\section{Second part: Listening to the patient's problem}

It is always important to understand patients' thoughts and feelings in order to help them feel calm and relaxed, as going to the dentist raises stress to most of the patients. At this part of the interview, the dentist can consider an initial inquiry into non-medical areas if appropriate [15,25]. Then the patient discusses his problem, explaining and describing symptoms or how he feels about treatment (active role), while the dentist listens carefully without speaking or interrupting (passive role), unless the patient has fully explained himself. This is a difficult situation for dentists because usually it is the professional who is active and the patient who is passive. In this part of the interview there is an apparent reversal of roles [17]. One study found that physicians typically wait only 23 seconds after a patient begins describing his main complain, before interrupting and redirecting the discussion [2]. This type of behavior besides making a bad impression makes it also difficult to really understand the person who is talking and giving information [13]. In general, listening skills are perhaps the most important of all of the verbal communication skills. Often listening is felt to have a passive quality. However, listening is one of the most active elements of verbal communication. The aim of active listening is to engage, facilitate and encourage the patient to speak by nodding or making open questions such as "what else?..." "When this started?..." etc [17].

It is then crucial to let patient explain his/her problem and encourage them by making open questions. Open questions are questions that cannot be answered by a simple yes or no and are more effective during an interview, because they allow patient to express their problem in detail [33]. The goal of such an interaction should be to fully understand and connect with the other person. An engaged way of listening, then usually comes naturally. In the opposite case there are some relevant steps to be followed. In the end, the dentist must give attention to what is being said and be able to reflect, clarify and rephrase patients' words. Finally, he must ensure that the patient is well understood. ${ }^{11}$ This part of the interview has also duration of no more than five minutes.

\section{Third part: Dentist's questioning about the problem}

In this part of the interview, the dentist plays the active role by addressing close type questions in order to clarify what the patient has already said. At this point, the dentist should encourage positively the patient on his good oral hygiene habits in order to make him feel comfort and trust. In the opposite, strict judgment on oral hygiene habits makes the patient defensive and reluctant to change [33]. 
Furthermore, in this part of the interview, questions on more personal details and habits can be addressed to the patient. The dental personnel should be extremely polite and discrete when asking delicate information on personal data (e.g "it would be necessary to have along with your mobile number the telephone number of your house" etc). All questions should be simple and easily understood (e.g. "have you checked this problem somewhere else too?"). It is crucial in this stage that only one question should be addressed each time. During the whole process, the dentist should learn to observe and examine the verbal and non verbal communication of the patient e.g. facial expressions, body movement and gestures, eye contact, posture, the tone of voice, the muscle tension and breathing, while distinguishing the useful information. In general, this part of the interview results in coming into conclusion for the personality and character of the patient. Practically, the dentist should be able to understand in which degree the patient fears or trusts him.

As a whole, in order for the dentist to arise empathy and understanding, he should use the technique of reflective listening and open body language. Reflective listening means that the dentist rephrases and repeats things that patient has already said by trying to move forward the conversation and retrieving more information [25]. Also the patient feels that he is heard, understood and well served [6]. For this reason, the dentist can use expressions like "Tell me more about it..." "Describe the pain for me..." "Let me see if I have understood this right..." [8]. The open body language e.g. arms uncrossed, standing with an open body shape or sitting on the edge of the seat and maintaining eye contact with the person talking, are basic rules. Furthermore while discussing with the patient the dentist should be aware of individual differences and specific body language signals. Also the tone of the voice, for example, should be different when a dentist is addressing a child than when a group of adults. Similarly, he should take into account the emotional state and cultural background of the person he is interacting with. The length of this part of the interview should also be five minutes.

\section{Fourth part: Clinical examination}

Before starting the clinical examination the dentist should explain in a simple thus detailed way what it shall happen next, e.g. "Now I shall look into your mouth and see what bothers you". The dentist should avoid at this stage any negative words and expressions no matter what he sees clinically. Before asking the patient to open his mouth he should be assured by the patient that he is sitting comfortable and that the head is in good position. e.g. "are you sitting ok? Should I fix the stand for the head? Furthermore a distance of $50 \mathrm{~cm}$ (one arm's length) should be kept between the face of the dentist and that of the patient in order to fulfill the oral examination without the patient to feel threatened. The questions made to the patient at this stage should be answered with a node. If needed more explanation the dentists should take out of the mouth his hands and tools so as to let the patient speak. No specific terms should be used while explaining the findings of the oral examination to the patient. Common mistakes that are being made at this stage of the interview and should be avoided, are the following: 1) discussion about irrelevant matters, 2) searching for tools, 3) disagreement with other staff members, 4) repeating questions already answered, 5) answering phone calls, 6) negative non verbal communication e.g. appearance, gestures, close body contact etc., and 7) no interaction with the patients' change of mood and body expressions [14,16,30]. In the end of the first appointment, the dentist should summarize in a simple language what has been said and get patient's agreement of this summary $[14,33]$.

\section{$2^{\text {nd }}$ appointment: Presentation and discussion of the treatment plan}

In the second appointment, the dental professional presents the possible treatment plans, describes each one of them, refers to benefits and also risks, helps patient decide which one fits best to his/hers case and in the end resumes what was decided [14,23]. Explaining is a fundamental aspect and an integral part of negotiating treatment options and health goals with patients. Explanations and dental health advice must be clear, concise and to the point $[17,35]$. The dentist should then reassure that the patient has understood what has been suggested, or the dental health education advice which has been given [17]. In this stage of the interview any discussion on the cost of treatment is made in a clear and written way. The dentist should remain constant in his decisions and be sure that the patient has fully understood the consequences of treatment and no treatment. All necessary explanations are given orally and written, within the dental office and not in the waiting room while the patient is leaving. The patient is being escorted to the door and leaves the office with a hand shake. This final part of the second appointment should last approximately fifteen minutes and the patient should leave knowing that all of his/hers concerns have been addressed [14,16,23]. 


\section{DISCUSSION}

Effective communication gives patients the sense that they have been heard and their concerns have been acknowledged, as well as respected, cared and understood. It also allows patients to express and reflect their feelings $[15,16]$. Thus dentists should always act within the jurisdiction of fairness, truthfulness and in the patient's best interest $[11,12]$. This means though that they should develop some abilities as referred to communication with their patients $[13,23,31]$.

Professionalism in the field of the private dental office is thus consisted of performing good dental practice together with the part of being an effective communicator. Such a professional is above all: 1) A good listener. Listening builds trust. Dentist should engage active listening by leaning forward and don't say a word. Also pause before replying so as to raise the self-esteem of the other person. It is also very important to speak slowly and avoid long monologues [2,12], 2) Empathic: showing empathy during the interview demonstrates good understanding of the patient's situation and feelings and ensures a trusting relationship between the dentist and the patient, 3) Honest: explaining the problem in a simple way with verbal language and terminology that the patient understands and telling the truth by not minimizing the impact of what is said but being also hopeful is another important value $[2,27,31]$, 4) Clear: The dentist should make clear explanations so that patients understand and interpret the message as originally intended. Not all the patients with the same diagnosis need the same level of detail in the information offered about their condition or treatment. Physicians should assess whether the patient desires or will be able to comprehend additional information [2]. Finally, it is always helpful and reassuring for the patient to explain to him what will happen before the dentist proceeds to the action [21,22,33,35].

Another important issue the dentists should be aware of when interviewing a new patient is the cultural diversity of the modern societies. Demographics are continually changing in the past years, because of immigration and also world's evolution [3]. For this reason, dentist needs to be able to communicate effectively with patients from a variety of racial, ethnic, linguistic, socioeconomic and cultural backgrounds. Culture and ethnicity affect communication in many ways. For instance, in some cultures personal questions asked during a dental examination can be perceived as offensive and invasion of privacy $[6,25,35]$. Of course, dentists should not let diversity be a barrier on providing good care. Understanding differences in cultural and ethnicities can help him give each patient the best available care [3] while being sensitive for differences at the same time.

In any case, the dentist should approach every patient with respect to the patient's autonomy and help him/her to make a treatment decision based on available information and honest advice. He should finally always remember that the stronger the relationship between dentist and patient, the more trust and faithfulness will ensure a long lasting cooperation.

\section{Conclusions}

- Dental procedure requires interpersonal communication which contributes to the therapeutic effects and also averts malpractice and judicial recriminations. Therefore, a good interpersonal relationship is a prerequisite for optimal dental/medical care.

- Interviewing a new dental patient requires usually two appointments during which the dentist needs to perform good communication skills both in verbal and non verbal ways. Empathy should constitute the main characteristic of both ways.

- Effective communication tips can be learned and practice by the dentist in order to promote trust and long-lasting professional relationships with the new patient.

\section{REFERENCES}

[1] Hannah A., Millichamp C.J., and Ayers KMS, A communication skills course for undergraduate dental students. J. Dent. Educ. 4, 971-977 (2004).

[2] Travaline J.M., Ruchinskas R., and D'Alonzo G.E., Patient-physician communication: Why and how. JAOA. 105, 13-18 (2005).

[3] Sifianou M., Politeness phenomena in England and Greece. A cross-cultural perspective. Clarendon press, Oxford University Press inc., New York (1992).

[4] Sondell, K. and Söderfeldt, B., Dentist-patient communication: A review of relevant models. 
Acta. Odon. Scand. 55, 116-126 (1997).

[5] Sherwin G., A more successful dental examination through better communication practices and self-understanding. Rev. Prog. 1, 2321-3485 (2013).

[6] Riga V. and Kossioni A., Body expression skills training in a communication course for dental students. Educ. Health. 27, 24-7 (2014).

[7] Piyush R., Atchanak R. and Avinash C., How can doctors improve their communication skills. J. Clin. Diag. Res. 9, JE01-JE04 (2015).

[8] Riga V. and Kossioni A., Communication between the dentist and the older dental patient. Hel. Hosp. Dent. 4, 23-28 (2011).

[9] Redhead P., How to read body language. Empower Solutions. pp.1-7 (2003).

[10] Giannidou S., Kostouli T. and Topitsoglou B., Communication between patient and dentist at the dental appointment. Hel. Stom. Review. 47, 75-84 (2003).

[11] Jacquot J., Trust in the dentist-patient relationship: A review. J Young Invest (2005). http://www.jyi.org/issue/trust-in-the-dentist-patient-relationship-a-review/

[12] Kurtz S.M., Doctor-patient communication: principles and practices. Can. J. Neurol. Sci. 29 (Suppl 2), 23-29 (2002).

[13] Dimitrius J.L. and Mazzarella M., Reading people. Thimari publ. 120-134, 250-265 (2001).

[14] Ha F.J. and Longnecker N., Doctor-patient communication: A review. Ochsner. J. 10, 38-43 (2010).

[15] Greenfield J.A., Health care communication. Healthcare. Comm. Review. 5, 1-7 (2005).

[16] Dorr Goold S. and Lipkin M., The doctor-patient relationship. Challenges, opportunities and strategies. J. Gen. Interl. Med. 14, 26-33 (1999).

[17] Freeman R., The psychology of dental patient care: Communicating effectively: some practical suggestions. Br. Dent. J. 187, 240-244 (1999).

[18] Misra S., Daly B., Dunne S., Millar B., Packer M. and Asimakopoulou K., Dentist-patient communication: what do patients and dentists remember following a consultation? Implications for patient compliance. Patient. Prefer. Adher. 7, 543-549 (2013).

[19] 19.Tongue J.R., Epps H.R. and Forese L.L., Communication skills for patient centered care: research-based, easily learned techniques for medical interviews that benefit orthopaedic surgeons and their patients. J. Bone Joint Surg. Am. 87, 652-658 (2005).

[20] Bellet P.S. and Maloney M.J., The importance of empathy as an interviewing skill in medicine. JAMA. 226, 1831-1832 (1991).

[21] Emmons Wener M., Schönwetter D.J. and Mazurat N., Developing new dental communication skills assessment tools by including patients and other stakeholders. J. Dent. Educ. 75, 15271541 (2011).

[22] Newburger L., 10 things patients want from a dental practice (2015). http://www.dentistryiq.com/articles/2015/03/10-things-patients-want-from-a-dental-practice.html

[23] Lypson M.L., Page A., Bernat C.K.and Haftel H.M., Patient-doctor communication: The fundamental skill of medical practice

[24] https://www.mededportal.org/icollaborative/resource/595 (2012).

[25] Ward J., 5 Ways to improve body language for better nursing. Nurse together. http://www.nursetogether.com/5-ways-improve-body-language-better-nursing-communication (2015)

[26] Lippincott W. and Wilkins G., Comprehensive dental assisting. Wolters Kluwer publ. pp. 400-411 (2012).

[27] Coleman H. and Burton J., Aspects of control in the dentist-patient relationship. Int. J. Sociol. Lang. 51, 75-104 (1985).

[28] Meyer E., Inside Dental Assisting. AEGIS. Communications. 10, 6-12 (2010).

[29] Holland K., Importance of body language in nursing communication. Nurse together. http://www.nursetogether.com/importance-body-language-nursing-communication (2014)

[30] Carlson N., Physiology of behavior. Pearson. pp.336 (2012). 
[31] American academy of family physicians. Division of medical education. Tips on building doctor patient relations. http://www.fmignet.aafp.org/ (2015).

[32] OSCE Home. Integrated Comprehensive Clinical \& Communication Skills Approach For Patient Interviews \& OSCE Exams. How To Improve My Communication Skills? Communication skills for healthcare professionals.

[33] http://www.oscehome.com/Communication-Skills.html (2013)

[34] Street R.L. Jr, Makoul G., Arora N.K. and Epstein R.M., How does communication heal, Patient Educ Couns. 74 (3), 295-301 (2009).

[35] Matulich B., How to do motivational interviewing. A guidebook. Kindle, $2^{\text {nd }}$ ed., (2013).

[36] Levin R.P., If you say it twice, script it. Dent. Econ. Oral Hyg. 88, 18-22 (1998).

[37] Wagner J., Arteaga S., D’Ambrosio J., Hodge C.E., Ioannidou E., Pfeiffer C.A., Yong L. and Reisine S., A patient-instructor program to promote dental students' Communication skills with diverse patients. J. Dent. Educ. 71, 1554-1560 (2007). 